Instrumental Achievements

\title{
Crystal Structure of Samarium Sesquisulfide, $\alpha-\mathrm{Sm}_{2} \mathrm{~S}_{3}$
}

\author{
Atsushi Aruga*, Sachiko Tsujimi** and Izumi NaKaI*** \\ * Department of Materials Science and Engineering, National Defense Academy, \\ Yokosuka, Kanagawa 239, Japan \\ **Analytical Chemistry 2nd, Tokushima Inst. of New Drug Res., Otsuka Pharmaceutical Co., Ltd., \\ Kawauchi, Tokushima 771-01, Japan \\ ***Department of Applied Chemistry, Faculty of Science, Science University of Tokyo, \\ Tokyo 162, Japan
}

The crystal structure of $\alpha-\mathrm{Ln}_{2} \mathrm{~S}_{3}$ type compounds ( $\mathrm{Ln}$ : rare earth elements) was first reported for $\alpha-\mathrm{Gd}_{2} \mathrm{~S}_{3}$ by Prewitt and Sleight. ${ }^{1}$ Though structure determinations were also carried out for lanthanum (Besançon and Laruelle $^{2}$ ), and neodymium (Eliseev et al. ${ }^{3}$ ), $R$ values were relatively high: $R=0.11$ or $R=0.131$, respectively. $\alpha-\mathrm{Ln}_{2} \mathrm{~S}_{3}$ is a low temperature phase and is stable at room temperature. Since $\alpha-\mathrm{Ln}_{2} \mathrm{~S}_{3}$ is the most basic compound among rare earth sulfides, the structure should be clearly understood. So a structure analysis of $\alpha-\mathrm{Sm}_{2} \mathrm{~S}_{3}$ was undertaken in order to obtain accurate structural information.

A selected single crystal was prepared by the silica tube method. A powder mixture of $\mathrm{Sm}$ and $\mathrm{S}$ in a molar ratio of $1: 1$ was sealed in an evacuated silica tube. This tube was heated for $30 \mathrm{~d}$ at $1100^{\circ} \mathrm{C}$. A single crystal of $0.08 \times 0.13 \times 0.13 \mathrm{~mm}$ was used for $\mathrm{X}$-ray structure determination. Crystal data and experimental conditions are given in Table 1 . Intensities of 1114 independent reflections were measured in the $2 \theta$ range of $1.5^{\circ}$ through $70^{\circ}$ using graphite-monochromated Mo $\mathrm{K}_{\alpha}$ radiation $(\lambda=0.70926 \AA)$. Lattice parameters were determined by a least-squares treatment of $2 \theta$ values of 25 reflections $\left(45^{\circ}<2 \theta<60^{\circ}\right)$. The intensity data were corrected for Lorentz and polarization factors; no absorption or extinction corrections were made. The crystal structure factor $F_{\mathrm{c}}$ was calculated using the atomic coordinates of isostructural $\alpha-\mathrm{La}_{2} \mathrm{~S}_{3}$ (Besançon and Laruelle $^{2}$ ) with overall temperature factors of 2.0 , the obtained $R$ value was $26.9 \%$ which was regarded as good for an initial model. Then, atomic parameters, scale and temperature factors were refined by the least-squares method. The final atomic positions and thermal parameters are given in Table 2 . The selected bond lengths and angles are listed in Table 3. Figure 1 is a view of the structure down the $b$ axis.

The structure consists of $\mathrm{Sm}(1) \mathrm{S}_{7}$ and $\mathrm{Sm}(2) \mathrm{S}_{8}$ polyhedra. $\operatorname{Sm}(2)$ is coordinated by eight sulfur atoms in a bicapped trigonal prism at an average distance of $2.914 \AA$ having two longer Sm-S distances: 3.077 and $3.042 \AA$. The trigonal prism stands along the $b$ axis.
Table 1 Crystal data and experimental conditions

Formula: $\alpha-\mathrm{Sm}_{2} \mathrm{~S}_{3}$
Formula weight $=396.90$
Crystal system: orthorhombic
Space group: $P_{n m a} \quad Z=4$
$a=7.376(1) \AA$
$b=3.9622(5) \AA$
$c=15.352(2) \AA$
$V=448.7 \AA^{3}$
$D_{\mathrm{x}}=5.88 \mathrm{~g} / \mathrm{cm}^{3}$
$\mu$ for Mo $\mathrm{K}_{\alpha}$ radiation $=271.7 \mathrm{~cm}^{-1}$
$R=0.051, \quad R w=0.068$
No. of reflections used $=1042$
Measurement: Rigaku AFC-5
Structure determination: Fourier method (GSFFR)
Program system: RFINE2
Refinement: full-matrix least-squares

Table 2 Atomic coordinates and temperature factors

\begin{tabular}{lcclc}
\hline & $x$ & $y$ & \multicolumn{1}{c}{$z$} & $B_{\mathrm{eq}} / \AA^{2}$ \\
\hline $\mathrm{Sm}(1)$ & $0.76572(9)$ & 0.25 & $0.54378(4)$ & 0.551 \\
$\mathrm{Sm}(2)$ & $0.14189(8)$ & 0.25 & $0.20400(4)$ & 0.486 \\
$\mathrm{~S}(1)$ & $0.0686(4)$ & 0.25 & $0.3915(2)$ & 0.579 \\
$\mathrm{~S}(2)$ & $0.1488(4)$ & 0.25 & $0.7812(2)$ & 0.481 \\
$\mathrm{~S}(3)$ & $0.3738(4)$ & 0.25 & $0.5669(2)$ & 0.601 \\
\hline
\end{tabular}

$B_{\mathrm{eq}}=4\left(\beta_{11} a^{2}+\beta_{22} b^{2}+\beta_{33} c^{2}\right) / 3$.

The Sm(1)S 7 polyhedron forms a distorted 7-coordinated octahedron at an average distance of $2.834 \AA$, with one longer Sm-S distance of $2.945 \AA$. These two coordination forms, though the former eightfold coordination is more common, are typical ones for the light rare earths up to dysprosium. The structure of $\alpha-\mathrm{Sm}_{2} \mathrm{~S}_{3}$ is a flat cell, whose thickness is about $4 \AA$, corresponding to the period of the $b$ axis. The height of the prisms 
Table 3 Selected bond lengths $(\AA)$ and bond angles $\left({ }^{\circ}\right)$

\begin{tabular}{|c|c|c|c|c|c|}
\hline \multicolumn{6}{|c|}{$\operatorname{Sm}(1) s_{7}$ polyhedron } \\
\hline $\sin (1)-s(1)$ & $2.945(3)$ & $\AA$ & $s(1)^{i}-\operatorname{sm}(1)-s(1)^{i v}$ & $95.37(8)^{\circ}$ & \\
\hline $\sin (1)-S(1)$ & $2.772(2) \times$ & $\times 2$ & $s(1)^{i}-\sin (1)-s(3)^{i v}$ & $75.07(8)$ & \\
\hline $\operatorname{Sm}(1)-S(2)$ & $2.822(3)$ & & $s(1)^{i v}-\operatorname{Sin}(1)-S(1)^{i v}$ & $91.24(9)$ & \\
\hline $\sin (1)-s(3)$ & $2.913(3)$ & & $s(1)^{W}-\operatorname{Sm}(1)-s(2) i$ & $80.92(8)$ & \\
\hline $\operatorname{Sm}(1)-S(3)$ & $2.806(2) \times$ & $\mathbf{x 2}$ & $S(1)^{i v}-\operatorname{Sm}(1)-s(3)^{i v}$ & $86.14(7)$ & $\times 2$ \\
\hline \multirow[t]{4}{*}{ Mean } & 2.834 & & $s(2)^{i i}-\sin (1)-s(3)^{i}$ & $65.21(9)$ & \\
\hline & & & $s(3)^{i}-\sin (1)-s(3)^{i v}$ & $73.13(8)$ & \\
\hline & & & $s(3)^{i v}-\operatorname{sm}(1)-s(3)^{i v}$ & $89.84(9)$ & \\
\hline & \multicolumn{3}{|c|}{$\operatorname{Sm}(2) S_{8}$ bicapped trigonal } & & \\
\hline $\sin (2)-s(1)$ & $3.042(3)$ & $\AA$ & $S(1)^{i}-S m(2)-S(2)^{i i i ~}$ & $77.05(7)^{\circ}$ & \\
\hline $\operatorname{Sm}(2)-S(1) *$ & $3.077(3)$ & & $s(1)^{i}-\sin (2)-s(2)^{i v}$ & $71.93(7)$ & \\
\hline $\sin (2)-S(2)^{\circ}$ & $2.777(2) \times$ & $\times 2$ & $s(1) i \mathrm{ii}-\mathrm{sm}(2)-s(2) \mathrm{iii}$ & $73.42(7)$ & \\
\hline $\operatorname{Sm}(2)-S(2)^{n}$ & $2.928(2) \times$ & $\mathbf{x 2}$ & $s(1) i i-\operatorname{sm}(2)-s(3) i i$ & $71.85(7)$ & \\
\hline $\operatorname{sm}(2)-S(3)$ & $2.893(2) \times$ & $\mathbf{x 2}$ & $s(2)$ iii $-\sin (2)-s(2)$ & $91.01(9)$ & \\
\hline \multirow[t]{5}{*}{ Mean } & 2.914 & & $s(2)^{i i i}-\sin (2)-s(2)^{\mathrm{N}}$ & $83.76(3)$ & \\
\hline & & & $s(2)^{i i i}-\sin (2)-s(3)^{i i i}$ & $81.04(7)$ & \\
\hline & $\cdot$ & & $s(2)^{i v}-\operatorname{Sm}(2)-s(2)^{i N}$ & $85.15(8)$ & \\
\hline & & & $s(2)^{i v}-\operatorname{sm}(2)-s(3)^{i i f}$ & $64.14(8)$ & \\
\hline & & & $s(3)^{i i i}-\sin (2)-s(3) i i i$ & $86.45(9)$ & \\
\hline
\end{tabular}

Symmetry code: i, $x, y, z$, ii, $1 / 2+x, 1 / 2-y, 1 / 2-z$; iii, $1 / 2-x$, $-y, 1 / 2+z ;$ iv $,-x, 1 / 2+y,-z . \times 2$ indicate that two crystallographically equivalent bonds or angles are involved in the same polyhedron.

determines the lattice period.

Each $\mathrm{Sm}(2) \mathrm{S}_{8}$ polyhedron is slightly zigzag, but linked to each other in the direction of the $a$ axis by sharing two triangle faces composed of one vertical edge of the prism and a cap of the polyhedron. Simultaneously, $\operatorname{Sm}(2) \mathbf{S}_{8}$ polyhedra are piled up along the $b$ axis by sharing two trigonal basal planes of the prism. As a result, the $\mathrm{Sm}(2) \mathrm{S}_{8}$ polyhedra form two-dimensional layers parallel to the $a b$ plane. The $\mathrm{Sm}(1)$ atom connects $\mathrm{Sm}(2) \mathrm{S}_{8}$

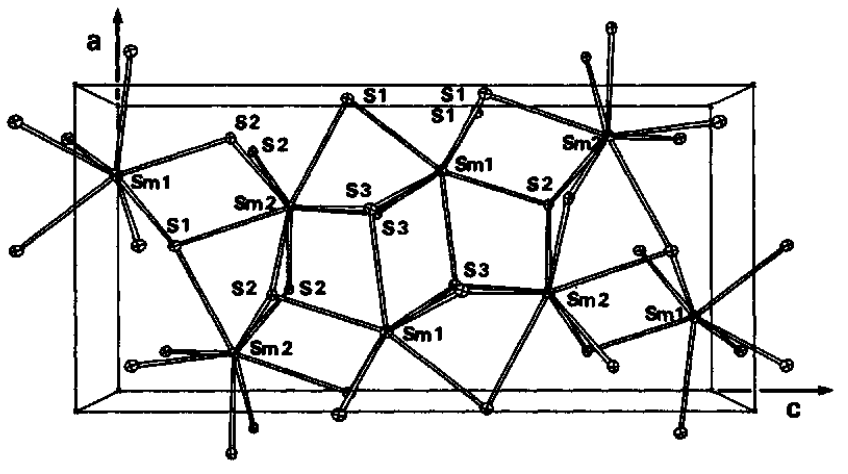

Fig. 1 ORTEP drawing of the crystal structure of $\alpha-\mathrm{Sm}_{2} \mathrm{~S}_{3}$ projected along the $b$ axis.

polyhedra by entering the 7-coordinated octahedral space between these layers. Each $\operatorname{Sm}(1) S_{7}$ polyhedron shares two pairs of opposite edges along the $a b$ plane. Namely, two layers of $\operatorname{Sm}(1) S_{7}$ and $\operatorname{Sm}(2) S_{8}$ alternately stack parallel to the $a b$ plane. The shape of $\operatorname{Sm}(2) S_{8}$ polyhedron leads to a small vacant space at the residual side of the prism. So the $\operatorname{Sm}(1) S_{7}$ polyhedron cannot share the faces with each other, but only the edges. It is clear that the structure is not a simple close-packed one, and the coordination polyhedra form a complex threedimension structure.

\section{References}

1. C. T. Prewitt and A. W. Sleight, Inorg. Chem., 7, 1090 (1968).

2. P. Besançon and P. Laruelle, Compt. Rendus., C268, 48 (1969).

3. A. A. Eliseev, S. I. Uspenskaya, A. A. Fedorov and V. A. Tolstova, Zh. Struk. Khim., 13, 77 (1972).

(Received October 2, 1995) (Accepted December 13, 1995) 